\title{
Correction to: Development of recombinase polymerase amplification assay for rapid detection of Meloidogyne incognita, M. javanica, M. arenaria, and M. enterolobii
}

\section{Yuliang Ju • Yu Lin • Guogen Yang • Huiping Wu •} Yuemin Pan

Published online: 30 January 2020

(C) Koninklijke Nederlandse Planteziektenkundige Vereniging 2020

Correction to: Eur J Plant Pathol (2019) 155:1155-1163 https://doi.org/10.1007/s10658-019-01844-6

Foundation number in "Acknowledgments", that is, "the National Natural Science Foundation of China (Grant No. 18234004)" should be read as "the National Natural Science Foundation of China (Grant No. 31801714 )".

The online version of the original article can be found at https://doi.org/10.1007/s10658-019-01844-6

Y. Ju $\cdot$ G. Yang $\cdot$ H. Wu $\cdot$ Y. Pan $(\bowtie)$

Key Laboratory of Biology and Sustainable Management of Plant Disease and Pests of Anhui Higher Education Institutes, Anhui Agricultural University, Hefei 230036, China e-mail: panyuemin2008@163.com

Y. Ju

e-mail: juyull@163.com

G. Yang

e-mail: yangguogen@ahau.edu.cn

H. Wu

e-mail: huiping.whp@163.com

Y. Lin

Tianjin Customs District of the People's Republic of China,

Tianjin 300456, China

e-mail: linyu010101@126.com 